\title{
RBF Interpolation Algorithm for FTS Tool Path Generation
}

\author{
Yahui Nie $\mathbb{D}^{1,2}$ Yinfei Du $\mathbb{C}^{3},{ }^{3}$ Zhuo Xu $\mathbb{D}^{1,2}$ Zimiao Zhang $\mathbb{D}^{1,2}$ and Yang $Q i \mathbb{D}^{1,2}$ \\ ${ }^{1}$ School of Mechanical Engineering, Tianjin University of Technology and Education, Tianjin 300222, China \\ ${ }^{2}$ Tianjin Key Laboratory of High-Speed Cutting \& Precision Machining, Tianjin University of Technology and Education, \\ Tianjin 300222, China \\ ${ }^{3}$ Tianjin Institute of Metrological Supervision and Testing, Tianjin 300192, China
}

Correspondence should be addressed to Yahui Nie; yahuinie@tute.edu.cn

Received 13 December 2020; Revised 10 January 2021; Accepted 23 January 2021; Published 2 February 2021

Academic Editor: Yi-Zhang Jiang

Copyright (c) 2021 Yahui Nie et al. This is an open access article distributed under the Creative Commons Attribution License, which permits unrestricted use, distribution, and reproduction in any medium, provided the original work is properly cited.

Freeform optics are defined as nonrotational symmetric optical surfaces in the manufacturing industry. Freeform optics are extensively applied to many areas in order to improve system performance. Fast tool servo (FTS) assisting single-point diamond turning technology has high application prospects in freeform optics machining. This paper discusses the interpolation algorithm for tool path generation of FTS through the application of a radial basis function (RBF) algorithm. For this purpose, a positive definite RBF with compact support was employed as the interpolant. The existence is mathematically proven. Numerical simulations were performed to compare the performances of the RBF algorithm and commonly used algorithms for satisfying the requirements of existence, smoothness, and accuracy. Machining experiments were also conducted to validate the applicability of the algorithm. The simulation results showed that the RBF interpolation algorithm outperformed other algorithms in terms of smoothness. The RBF algorithm also provided the highest interpolation accuracy. Furthermore, the RBF interpolation algorithm exhibited the highest accuracy for error distribution, with large errors distributed mainly in transition areas. The machining results were also in general agreement with the simulation results although obvious practical errors were observed. Overall, RBF interpolation can provide higher accuracy and better smoothness in the tool path generation of FTS.

\section{Introduction}

Freeform optics are extensively applied in various areas, such as aerospace, energy, and life science, to improve system performance and reduce overall system complexity and system weight $[1,2]$. Duerr et al. used freeform optics to realize superior imaging performance for applications with high aspect ratio [3] and also used freeform optics to perform static and dynamic laser beam shaping systems [4]. Freeform optics are widely used to shape light according to a desired target distribution given target uniformity when light emitting diodes are routinely used for general and specialized lighting tasks such as automotive headlights, streetlights, luminaires, and medical illuminators [5]. Helmet-mounted displays (HMDs) employ three freeform mirrors to provide an image source for generating information images that are optically presented to the user's eyes, allowing pilots to focus only on data displayed on the goggles and freeing them from complex instrumentation operations [6]. Optical freeform surfaces can achieve good optical performance, but it is difficult to fabricate freeform optics with high quality and high efficiency due to the complexity of surfaces and high requirements of profile accuracy and roughness. Thus, ultraprecision machining is always employed to fabricate freeform surfaces.

Freeform optics can be fabricated using various techniques such as micromilling, raster-cutting or fly cutting, profile and form grinding, and fast tool servo (FTS) [7]. FTS assisting single-point diamond turning has very good application prospects in freeform optics for improving machining accuracy, surface roughness, and machining speed. FTS can be used to machine brittle materials, such as silicon and germanium. Thus, many researchers employ FTS to fabricate specially designed freeform surfaces. Kim fabricated freeform surfaces that are frequently demanded for the construction of optical imaging systems using FTS [8]. Schieiding machined micro- 
optical lens-arrays on a steep curved substrated by use of a voice coil FTS [9]. Tian et al. used ultraprecision diamond turning machining technology based on an FTS system to realize 3D surface generation of a rear-view mirror, which is a typical optical freeform surface [10]. Li et al. fabricated a rectangular freeform surface on germanium using FTS, satisfying designing targets of the freeform surface [11].

A typical machine configuration for FTS machining is shown in Figure 1. The FTS is mounted on the $Z$ axis carriage as an auxiliary axis $\left(Z^{\prime}\right)$ [12]. In FTS machining, the cutting tool is mounted on an auxiliary axis $\left(Z^{\prime}\right)$, which is optimized to perform sinewave type oscillations as the $C$ axis rotates while the $X$ and $Z$ axes produce linear feed [13].

With the maturity of FTS equipment, many kinds of commercial FTS with various strokes and bandwidths have already been designed to fabricate different kinds of freeform optics [14-16]. In recent years, the study of FTS has been focused on machining methods and techniques instead of hardware [17-19].

The procedure of tool path generation of FTS can be described as follows [20]:

(1) Tool compensation: cutting tool compensation should be calculated because surfaces have mutative curvature and different cutting points exist between the tool and workpiece [21]. As a result, the original cutter contact point will change into cutter location point. Then, the data used to describe freeform optics will change from gridded data into scattered data.

(2) Regeneration of freeform surfaces: the scattered data should be interpolated into gridded data to guarantee the online interpolation speed and avoid frequent reciprocation of the $X, C$, and $Z$ axes.

(3) Surface decomposition: the original freeform surfaces can be decomposed into two parts-a rotational symmetric surface (generated by the $X, C$, and $Z$ axes) and a fully freeform surface (generated by the $X, C$, and $Z^{\prime}$ axes). Surface decomposition can reinforce the machine's ability to generate larger sagittal height of freeform surfaces.

(4) Online interpolation of cutting points: the cutting points are calculated online based on the interpolation algorithm. Then, the movements of FTS can be obtained based on the current position of the $C$ and $X$ axes.

Based on the procedure of FTS machining, it can be concluded that the interpolation algorithm is very important. An algorithm with high accuracy and calculation speed is required. However, the existing algorithms are not sufficient in speed and accuracy. This study focused on the tool path generation technique of FTS. An interpolation algorithm with higher accuracy in the tool path generation technique was investigated to improve the machining profile accuracy of FTS.

The interpolation algorithm in the tool path generation of FTS affects the machining accuracy of freeform optics. The interpolation algorithm totally decides the accuracy of the described surface in the computer, controlling the motion of the four axes. The requirements of interpolation in tool path generation of FTS are as follows. Firstly, the interpolation algorithm should have the ability to obtain a determined solution at any point on the surface (existence). Secondly, the interpolation function should be second-order continuous to guarantee smoothness of surface (smoothness). Finally, interpolation errors should be below the subnanometer scale in the $Z$-axis (accuracy).

$\mathrm{Hu}$ et al. used the four-point mean value interpolator for FTS diamond turning to improve the surface accuracy of the freeform surface and meet the real-time requirement compared with nonuniform rational B-splines (NURBS) [22]. However, neither the accuracy nor the smoothness requirement could be fulfilled. Li and Zhou both used Hermite interpolators to regenerate the surface $[11,23]$. However, all points on the surface could not achieve a determined solution due to the characteristics of the Hermite interpolant. Nie used an optimized Shepard interpolator for the tool path generation of FTS [24]. However, large amounts of surface data are required to satisfy accuracy requirements, and distortion also occurs in the regenerated surface. Different kinds of interpolation algorithms are applied to realize regeneration of the freeform surface in the tool path generation of FTS. However, the requirements of the FTS interpolation algorithm have not been fulfilled.

In this study, radial basis function (RBF) interpolation was applied as the interpolation algorithm in the tool path generation of FTS to process freeform optics. RBF interpolation was adopted because it can satisfy the three requirements of FTS. Firstly, it has the advantage of high accuracy, improving machining accuracy. Secondly, it has the advantage of fast convergence, which facilitates the acquisition of a determinate solution at any point on the surface. Thirdly, most RBFs are second-order differentials, ensuring high smoothness of the surface approximated by RBF interpolation. The better smoothness further ensures better surface roughness.

\section{Optimized Radial Basis Function Interpolation}

2.1. Interpolation Model. The interpolation algorithm for the tool path generation of FTS should be classified in the multivariate scattered data interpolation problem. The problem of interpolation for FTS can be described as follows: a set of $3 \mathrm{D}$ data $\left\{\mathbf{p}_{j}=\left[x_{j}, y_{j}, z_{j}\right\}_{j=1,2, \ldots, n}\right.$ can be obtained based on equations of the freeform surface to be processed. Here, we define $\left\{\mathbf{p}_{j}=\left[x_{j}, y_{j}\right]\right\}_{j=1,2, \ldots, n}$ as sampling points and $\left\{z_{j}\right\}_{j=1,2, \ldots, n}$ as sagittal height. A function $f(\mathbf{p})$ is desired with centers at the sampling points $\left\{\mathbf{p}_{j}\right\}$ that interpolates the sagittal height $\left\{z_{j}\right\}$. Thus, $f(\mathbf{p})$ is such that

$$
z_{j}=f\left(\mathbf{p}_{j}\right)=f\left(x_{j}, y_{j}\right), \quad j=1,2,3, \ldots, n .
$$

Furthermore, the problem can be described into a general mathematical problem, which can be described as follows: $\lambda j$ is a linear function in $C\left(R^{2}\right)$; for the given points $f j \in R, j=1,2, \ldots, n$, a function $f(\mathbf{p})$ is desired to satisfy the following equation: 


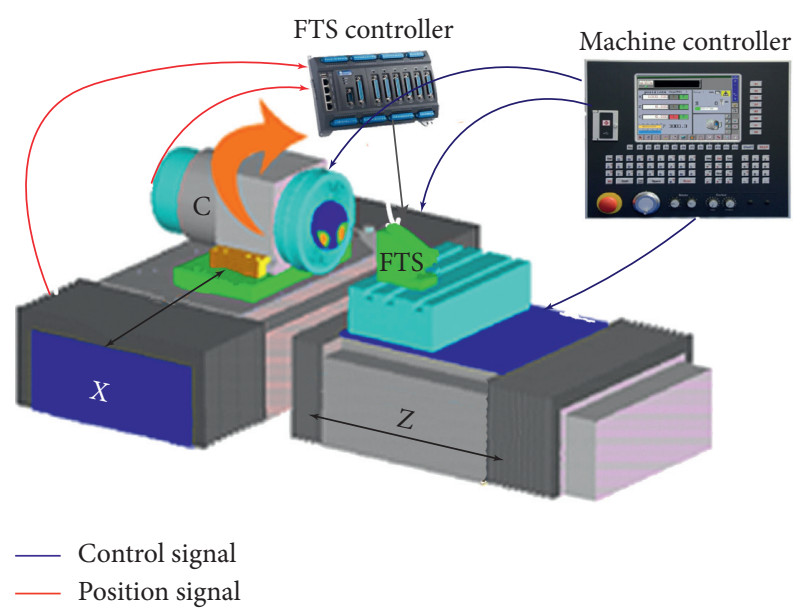

FIgURE 1: Typical configuration of fast tool servo machining.

$$
\lambda_{j} \cdot f(\mathbf{p})=f_{j}, \quad j=1,2,3, \ldots, n .
$$

In this equation, $f(\mathbf{p})$ is the interpolant of the interpolation algorithm.

2.2. Radial Basis Function Interpolation. The RBF $\phi\left(\mathbf{p}_{j}\right)$ is a simple multivariate function. RBF interpolation is more attractive than methods that require complex preprocessing because the structure of RBF is simple. RBF can be simply represented by a computer. Thus, $\mathrm{RBF}$ interpolation is commonly used in signal processing, photogrammetry, digital terrain modeling, and so on $[25,26]$. RBF interpolation for scattered data can be described as follows.

An $\operatorname{RBF} \phi$, which is a mapping from $\mathbf{R}^{+}$to $\mathbf{R}$, is given. For scattered data $\left\{x_{j}, y_{j}, z_{j}\right\}_{j=1,2, \ldots, n} \in R^{2} \otimes R$, a function $f(\mathbf{p})=$ $\sum f_{j} \phi\left(\left\|\mathbf{p}-\mathbf{p}_{j}\right\|\right)$ is desired. The function $f(\mathbf{p})$ should satisfy

$$
f_{k}=\sum a_{j} \phi\left(\left\|\mathbf{p}_{k}-\mathbf{p}_{j}\right\|\right)
$$

Equation (3) is the interpolation function. $\left\{a_{j}\right\}_{j=1,2,3, \ldots, n}$ are pending coefficients, which are calculated through $\left\{x_{j}, y_{j}, z_{j}\right\}$ $j=1,2,3, \ldots, n$. Then, satisfying interpolation results will be obtained after a proper $\operatorname{RBF} \phi\left(\left\|\mathbf{p}-\mathbf{p}_{j}\right\|\right)$ is selected.

$\mathrm{RBF}$ are widely studied. Gaussian interpolant, Kriging interpolant, multiquadric interpolant, and inverse multiquadric are favorable RBFs. A positive definite RBF with compact support is constructed using convolution to satisfy the requirements of FTS:

$$
\phi\left(r_{i, j}\right)=-r_{i, j}{ }^{3}+4 r_{i, j}{ }^{2}+c,
$$

where $r_{i, j}=\left|x_{i}-x_{j}\right|+\left|y_{i}-y_{j}\right|$. The real-time requirement should be fulfilled by employing a controller with hardware multiplier considering the multiplication in equation (4). Obviously, equation (4) is a second-order differential function, through which the smoothness of interpolated surfaces can be fulfilled.

\subsection{RBF Interpolation for Tool Path Generation of FTS.} The cutter location points (CLPs) or the machining points in the machine controller are all scattered data, which cannot be described by gridded data. In the FTS interpolation, a localized interpolation is adopted considering the realization of the interpolation algorithm in the controller. For the current machining point $\mathbf{p}(x, y)$, we use RBF interpolation to neighbor $n$ points (generally $n=4,8,10)$ to obtain the $z$ value. Then, FTS is driven to generate the movement of $z$. A freeform surface will be generated after all the machining points are interpolated. Interpolation to the chosen $n$ neighbor points means that the current machining point and its neighbor points can be described by a function (2).

In this paper, $\mathrm{RBF}$ interpolation to $n$ neighbor points $\left\{\mathbf{p}_{i}\left(x_{i}, y_{i}\right)\right\} i=1,2, \ldots, n$ for the current machining point $\mathbf{p}(x, y)$ uses the positive definite RBF with compact support as the interpolant (4). The pending coefficients in equation (3) can be calculated via the following linear system:

$$
\left[a_{1}, a_{2}, \ldots, a_{n}\right]\left[\begin{array}{cccc}
\phi_{1,1} & \phi_{2,1} & \cdots & \phi_{n, 1} \\
\phi_{1,2} & \phi_{2,2} & \cdots & \phi_{n, 2} \\
\vdots & \vdots & \ddots & \vdots \\
\phi_{1, n} & \phi_{2, n} & \cdots & \phi_{n, n}
\end{array}\right]=\left[\begin{array}{c}
f_{1} \\
f_{2} \\
\vdots \\
f_{n}
\end{array}\right]
$$

where $\phi_{i, j}=\phi\left(\left\|\mathbf{p}_{i}-\mathbf{p}_{j}\right\|\right)$. Equation (5) is solvable if $\phi\left(\| \mathbf{p}_{i}-\right.$ $\left.\mathbf{p}_{j} \|\right)$ is a positive definite matrix.

Then, an interpolation function for the current machining point can be obtained as follows:

$$
f(\mathbf{p})=\sum_{j=1, n} a_{j} \phi\left(\left\|\mathbf{p}-\mathbf{p}_{j}\right\|\right),
$$

where $\phi\left(\left\|\mathbf{p}_{i}-\mathbf{p}_{j}\right\|\right)$ is a positive definite matrix as long as $\mathbf{p}_{i} \neq \mathbf{p}_{j}$. In our interpolation situation, $\mathbf{p}_{i} \neq \mathbf{p}_{j}$ will be satisfied because the original data do not contain repeated points. Thus, problem (2) has a solution for any interpolation point [27]. In this manner, the existence requirement of FTS is satisfied by the RBF interpolation algorithm. Thus, the RBF interpolation algorithm can be employed in the tool path generation of FTS to regenerate surfaces.

\section{Experiments of Radial Basis Function Interpolation}

3.1. Simulation Experiments. Simulation experiments were carried out to verify that the RBF interpolation algorithm has the advantage of high accuracy and good smoothness. Three interpolation algorithms commonly used in the industrial area (Hermite interpolation algorithm, four-point mean interpolation algorithm, and Shepard interpolation algorithm) were employed to compare the performance of different algorithms.

3.2. Conditions of Simulation Experiments. Simulation experiments are performed in MATLAB software. The simulated workpiece is a lens array, which is a typical workpiece for evaluating the performance of FTS (Figure 2). The lens array characteristics and the simulation parameters are described in Table 1. 


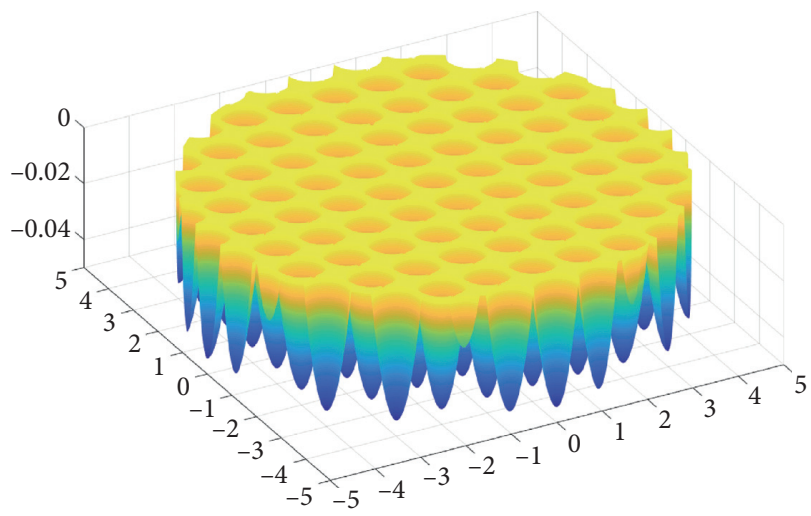

Partial enlargement view of one lens

FIGURE 2: Freeform optics simulated in experiments.

TABLE 1: Parameters of simulated workpiece.

\begin{tabular}{lc}
\hline Diameter of workpiece & $10 \mathrm{~mm}$ \\
\hline Aperture of small lens & $1 \mathrm{~mm}$ \\
Pitch between two lenses & $1 \mathrm{~mm}$ \\
Radius of small lenses & $2.84 \mathrm{~mm}$ \\
Sagittal height of lenses & $-44.4 \mu \mathrm{m}$ \\
\hline
\end{tabular}

3.3. Comparison of Smoothness. In the experiments of smoothness, the original data quantity is 4800 . The original data describe freeform surfaces to be processed. The interval between two points on the surface is $0.125 \mathrm{~mm}$. Although the intensity of data in this experiment is rarely used in actual fabrication, it can clearly show the smoothness of the four surfaces generated by different interpolation algorithms. Then, analysis can be performed based on the simulation results. The four surfaces obtained using the four different algorithms are shown in Figure 3.

Curves of integral smoothness variation versus data amount are shown in Figure 4. The simulation experiment results of the four interpolation algorithms show the following. (1) The RBF interpolation algorithm performs the best in terms of smoothness, followed by the Shepard interpolation algorithm. The four-point mean interpolation algorithm performs the worst. (2) The surface generated by the Hermite interpolation algorithm has good smoothness, but some points on the surface cannot be calculated due to the characteristic of the Lagrange interpolation function. This is because in multidimensional interpolation, any interpolation basis function cannot satisfy the HARR condition. Thus, some interpolation basis functions (such as Lagrange and Bernstein), which perform well in dimensional interpolation, will not provide interpolated solutions at all points. Most time solutions of most points can be calculated, but the solutions may not be stable [28]. Points without calculated value are shown in Figure 3 as blank areas. (3) The four-point mean algorithm is very simple, but its smoothness is the worst. Figure 3 shows obvious steps on the surface generated by the four-point mean algorithm. (4) The Shepard interpolation algorithm performs well in terms of smoothness, but distortions occurred at peaks of each small mirror (Figure 3 ). The distortion of small mirrors are caused by the duck egg effect of the Shepard interpolation algorithm [29]. The distortion may affect the performance of freeform optics. Moreover, small steps can be observed in its interpolation figure. Thus, according to the simulation results, the RBF interpolation algorithm satisfies the requirements of FTS with best results and is thus a more suitable option for tool path generation of FTS.

3.4. Comparison of Profile Accuracy. In order to compare the actual interpolation accuracy of the four algorithms, original scattered data with different densities were simulated. In the simulation experiments, the mean errors of the interpolated surface were adopted to evaluate profile accuracy, which is described as follows:

$$
\text { Error }_{t}=\frac{\sum_{i=1}^{n}\left|f\left(p_{i}\right)-g\left(p_{i}\right)\right|}{n},
$$

where $f(\mathbf{p})$ is the interpolation value and $g(\mathbf{p})$ is the actual value calculated according to the formula of the designed freeform surfaces. Then, simulation results of profile accuracy were obtained, as shown in Table 2. Figure 5 shows the interpolation accuracy variation versus data densities.

The simulation experiment showed the following. (1) The interpolation accuracy decreased with increasing data amount for the Shepard interpolation algorithm, Hermite interpolation algorithm, and RBF interpolation algorithm. However, the interpolation accuracy of fourpoint mean algorithm does not obey this law, and its accuracy is lowest among four interpolation algorithms. (2) The RBF interpolation showed the highest interpolation accuracy. Furthermore, with increasing data amount, errors of the RBF interpolation decreased at a faster rate than the other two algorithms (Hermite interpolation algorithm and four-point mean interpolation algorithm). (3) The interpolation accuracy was achieved at the nano level, fulfilling the accuracy requirement of ultraprecision machining. Considering machine accuracy, intervals between two points ranging from $0.01 \mathrm{~mm}$ to $0.02 \mathrm{~mm}$ are recommended for the RBF interpolation algorithm. Intervals between two points are recommended to be smaller than $0.01 \mathrm{~mm}$ for the Shepard algorithm. 


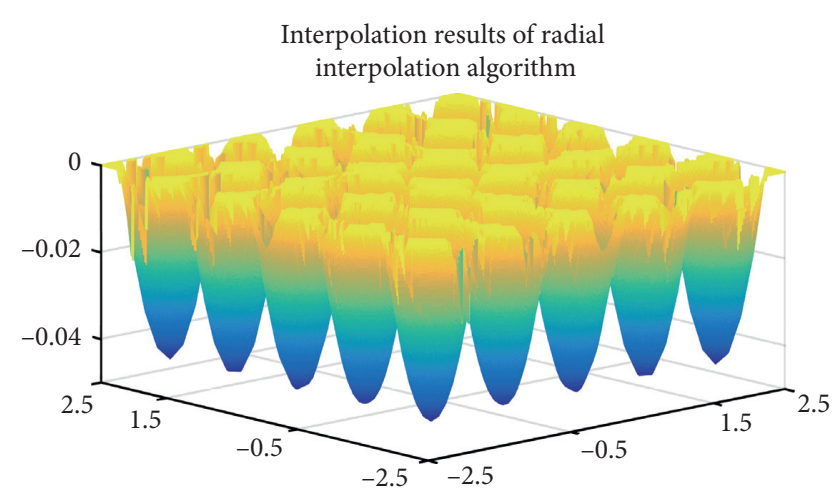

Interpolation results of Sheperd interpolation algorithm

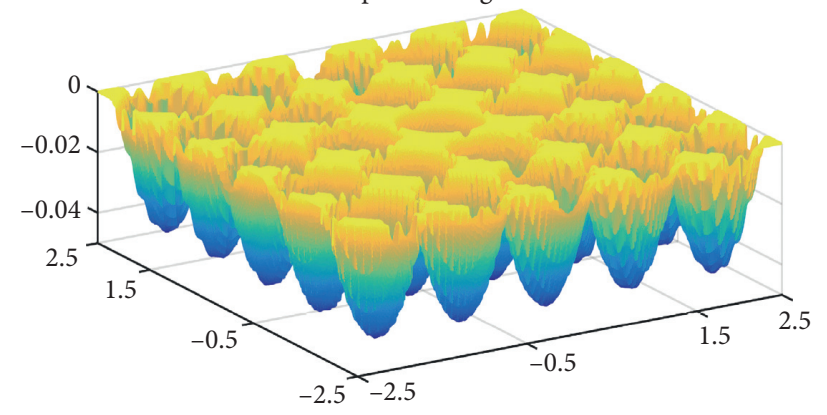

Interpolation results of four-point mean interpolation algorithm

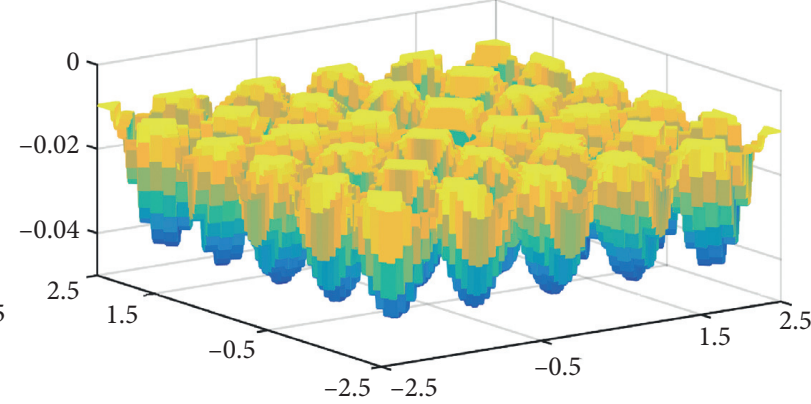

Interpolation results of Hermite interpolation algorithm

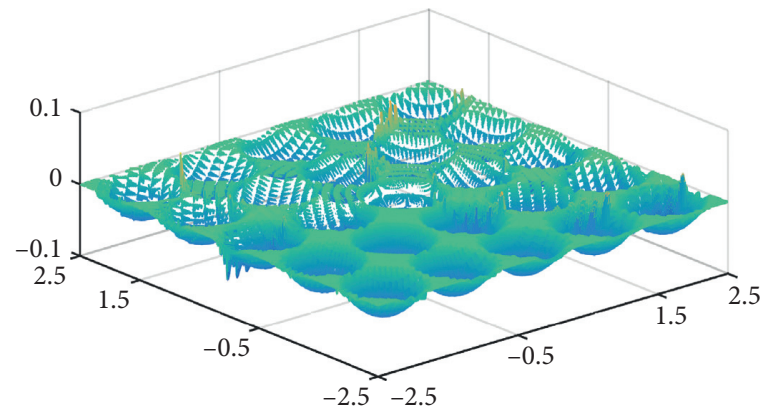

FIgURE 3: Simulation experiment results of four interpolation algorithms.

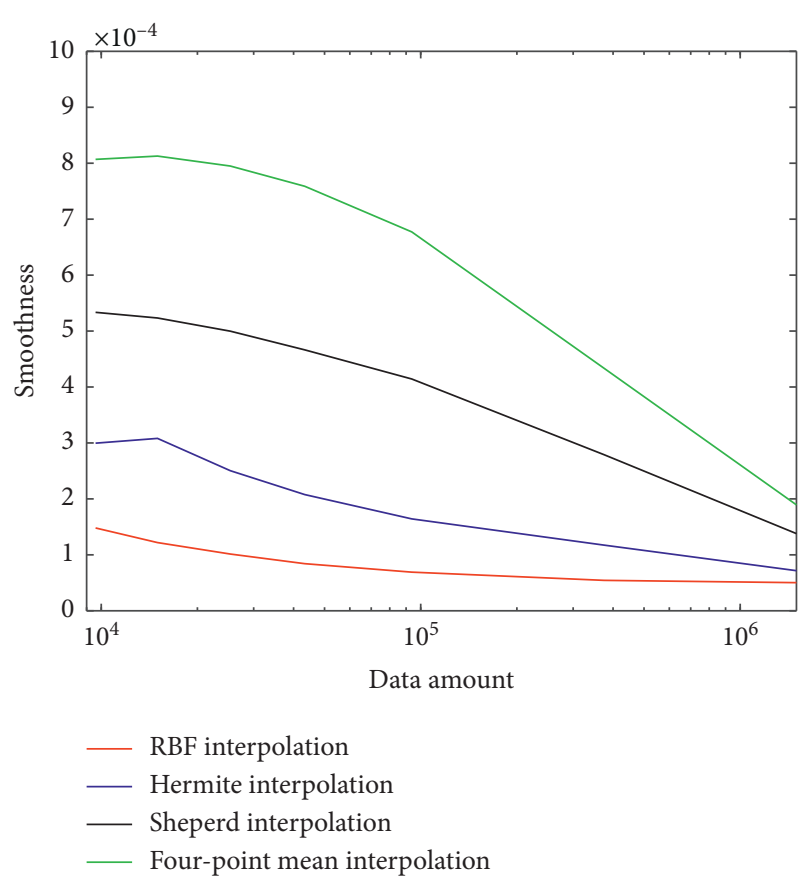

FIGURE 4: Integral smoothness variation curve versus data amount.

3.5. Error Distribution of Four Algorithms. Freeform optics processed by FTS should achieve a specific function. Thus, error distribution is an important factor to judge whether a specific interpolation algorithm is suitable. The designed function will be severely affected if interpolation errors are mainly distributed at functional areas of freeform optics. Error distributions of the four algorithms were simulated in our experiment. Intervals between two neighbor points were $0.04 \mathrm{~mm}$ in the original scattered data, which is recommended as a preferable interval by engineers and researchers [9]. The error distributions of the four algorithms are shown in Figure 6.

The error distribution simulation results show that errors are distributed on the whole surface for the four algorithms. Large errors are mainly distributed at the edges of each small lens in the Shepard and especially the $\mathrm{RBF}$ interpolation algorithms. Large errors of the fourpoint mean and Hermite interpolation algorithms are mainly distributed in each small lens. Errors of these two algorithms have the same changing trend with the sagittal height, i.e., the higher the sagittal height, the larger the errors. Generally, transition areas of freeform optics (edges in our experiments) need not be processed for high accuracy because most mechanical processing techniques, especially FTS technique, will smoothen transition areas to avoid resonance in the linear axis. After the smoothening, the accuracy of the original surface is reduced. Based on the simulation experiment of error distribution, the RBF interpolation algorithm exhibited the highest accuracy, with large errors distributed mainly in transition areas. Thus, according to simulation results and practical processing requirements, RBF interpolation will provide better performance.

According to the simulation experiments, it can be concluded that RBF performs the best in satisfying FTS requirements of existence, smoothness, and accuracy. 
TABLE 2: Accuracy value of four interpolation algorithms with different intervals.

\begin{tabular}{lccccc}
\hline \multirow{2}{*}{ Accuracy $(\mathrm{mm})$} & \multicolumn{5}{c}{ Interval (amount) } \\
& $0.125 \mathrm{~mm}(9600)$ & $0.08 \mathrm{~mm}(25350)$ & $0.04 \mathrm{~mm}(93750)$ & $0.02 \mathrm{~mm}(375000)$ & $0.01 \mathrm{~mm}(1500000)$ \\
\hline RBF & $8.6338 e-04$ & $3.5278 e-04$ & $1.0250 e-04$ & $2.5318 e-05$ & $7.2162 e-06$ \\
Hermite & 0.0011 & $6.0755 e-04$ & $3.1390 e-04$ & $1.5349 e-04$ & $7.9757 e-05$ \\
Four-point mean & 0.0059 & 0.0045 & 0.0034 & 0.0063 & 0.0044 \\
Shepard & 0.0015 & $8.5607 e-04$ & $4.3173 e-04$ & $2.0430 e-04$ & $1.0157 e-04$ \\
\hline
\end{tabular}

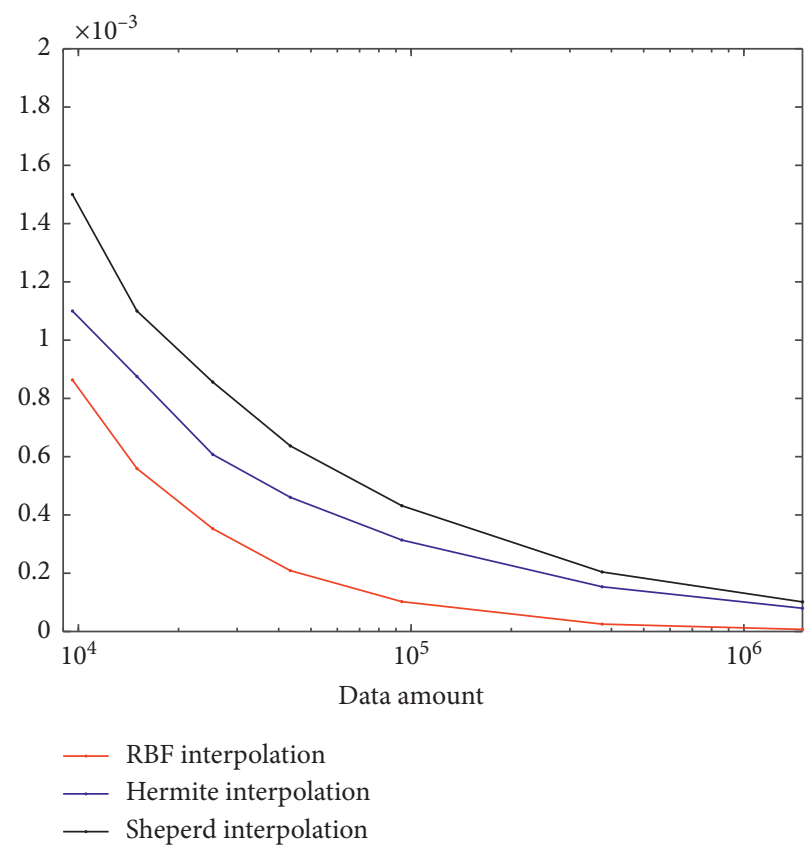

FIGURE 5: Profile accuracy variation curve versus data amount.

3.6. Machining Experiment. Machining experiments were carried out on a commercial ultraprecision lathe (UPL250), configured with an FTS (NFTS6000) as the $Z^{\prime}$ axis. In order to compare the accuracy of the four interpolation algorithms, the same parameters of the workpiece were adopted, as shown in Table 1. Machining parameters are described in Table 3. The number of original scattered data was 9370, and intervals between two points were $0.04 \mathrm{~mm}$.

Considering that accurate measurement of freeform optics is still an unresolved issue, each small lens on the processed workpiece was measured with the Taylor Hobson profiler in our experiment. In the machining experiment, the edges of the lenselets will be preprocessed by a new smoothing algorithm proposed by us, and thus the edges of the lenselet will not affect the machining and measuring results. Average measurement results of all small lenses on each workpiece are shown in Table 4 , and the measurement results of a small lens at the same spot on each workpiece are shown in Figure 7.
Based on actual processing experiment, it can be concluded that (1) the profile accuracy of the freeform optics processed by FTS can be improved by improving interpolation accuracy; different profile accuracies will be obtained based on various interpolation algorithms; (2) the RBF interpolation algorithm can provide the highest accuracy; (3) the Shepard and Hermite interpolation algorithms provide good profile accuracy, but the error value increases with increasing scallop height; and (4) the four-point mean interpolation algorithm has the lowest profile accuracy. Nevertheless, considering practical processing applications, it can be used in applications with low accuracy requirement for its simplicity.

The machining experiment results agree with simulation results. Considering other systematic errors (errors of fabrication of the three axis, errors of displacements, errors of heat, and so on), it is not surprising that the actual machining errors are larger than the simulation value. 


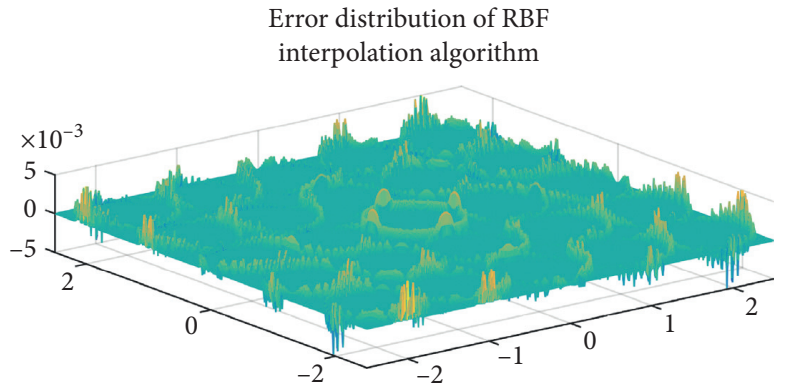

Error distribution of Sheperd interpolation algorithm

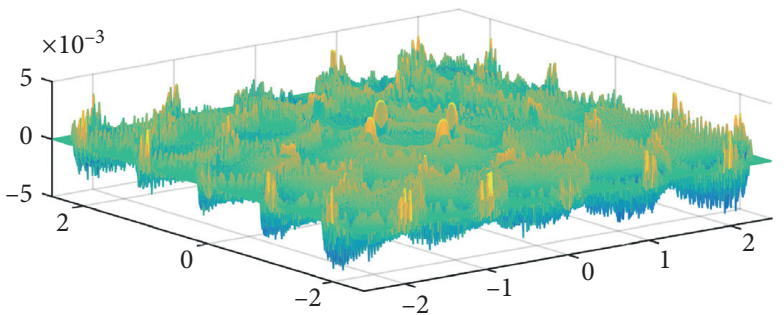

Error distribution of four-point mean interpolation algorithm

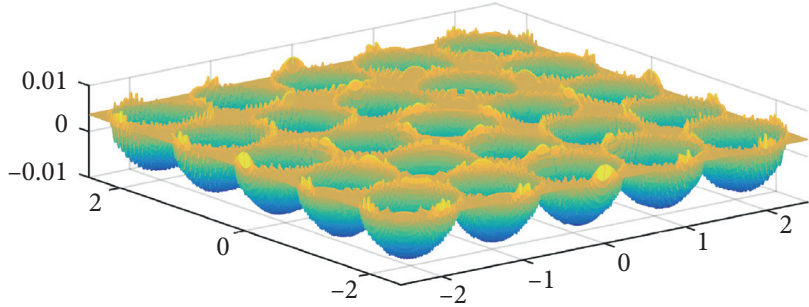

Error distribution of Hermite interpolation algorithm

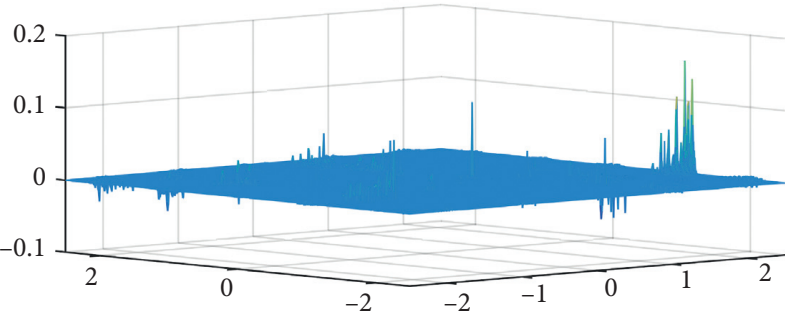

FIgURE 6: Error distributions of four interpolation algorithms.

TABLE 3: Machining parameters.

Spindle speed

Depth of cut $800 \mathrm{rpm}$

Tool radius

TABLE 4: Measurement results of profile accuracy of four interpolation algorithms.

\begin{tabular}{lcccc}
\hline Algorithm & FPM $(\mathrm{nm})$ & Hermite $(\mathrm{nm})$ & Shepard $(\mathrm{nm})$ & RBF $(\mathrm{nm})$ \\
\hline Profile accuracy (mean) & 682.2 & 225.6 & 209.8 & 103.2 \\
Roughness & 5.84 & 5.58 & 5 & 4.8 \\
\hline
\end{tabular}

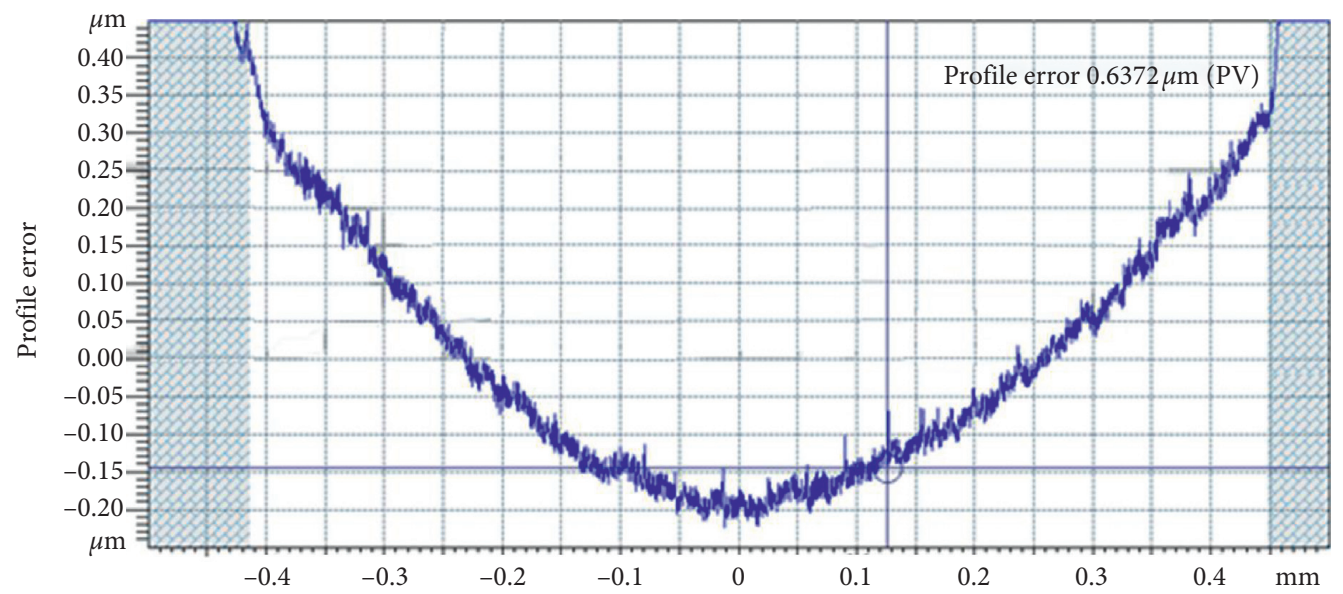

(a)

FIgURE 7: Continued. 


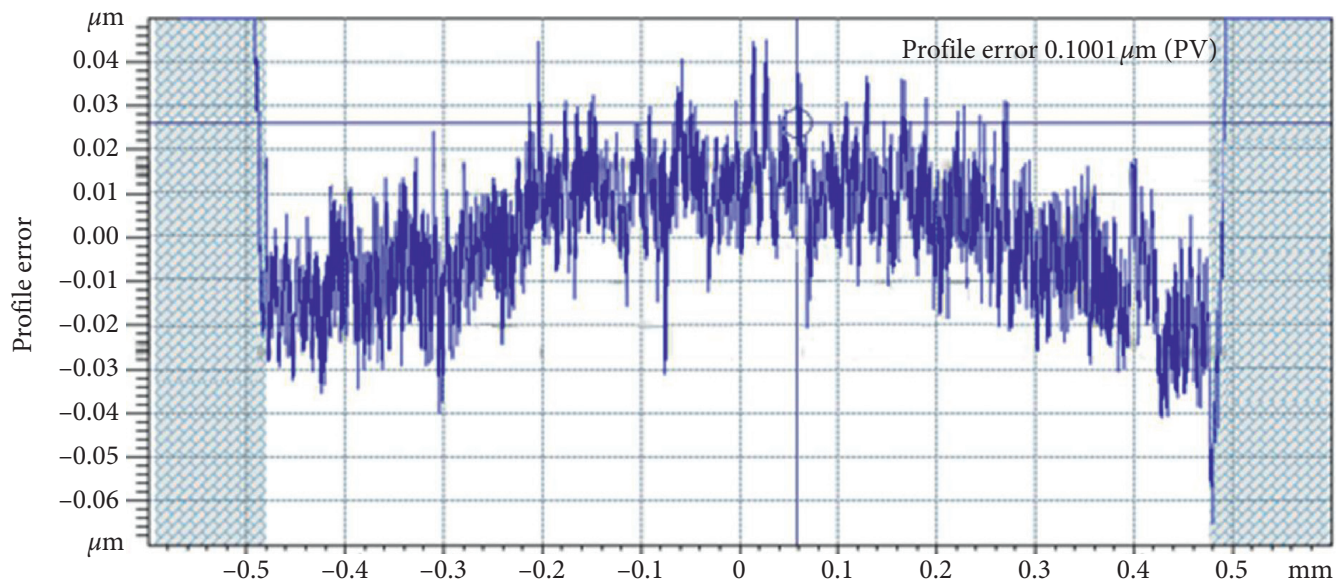

(b)

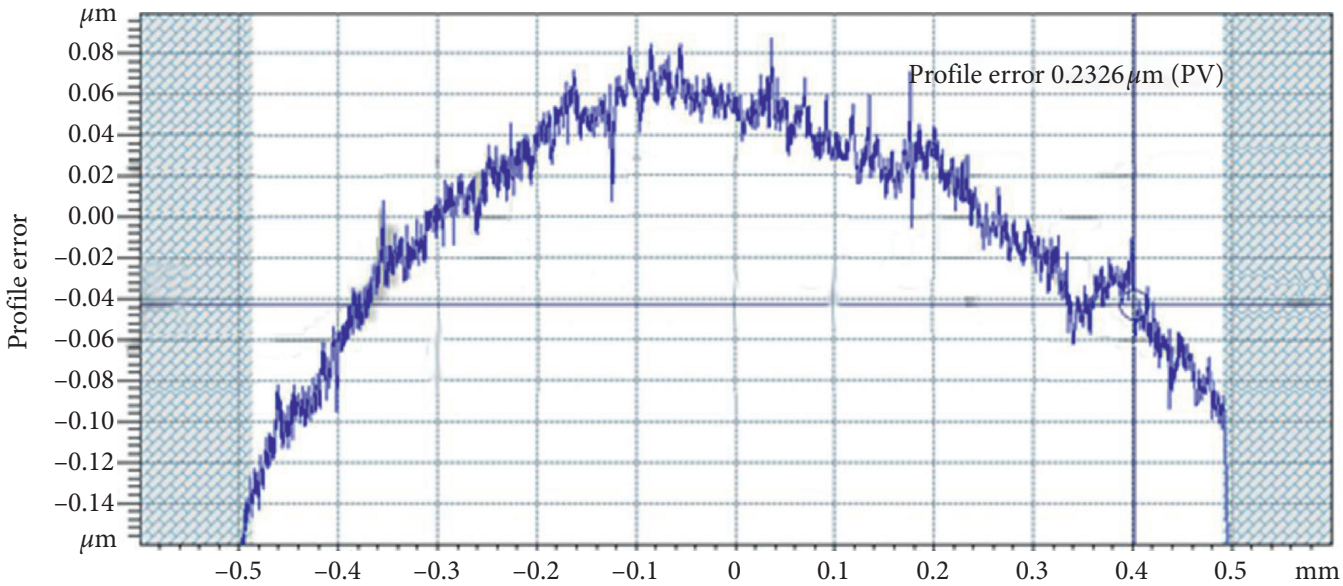

(c)

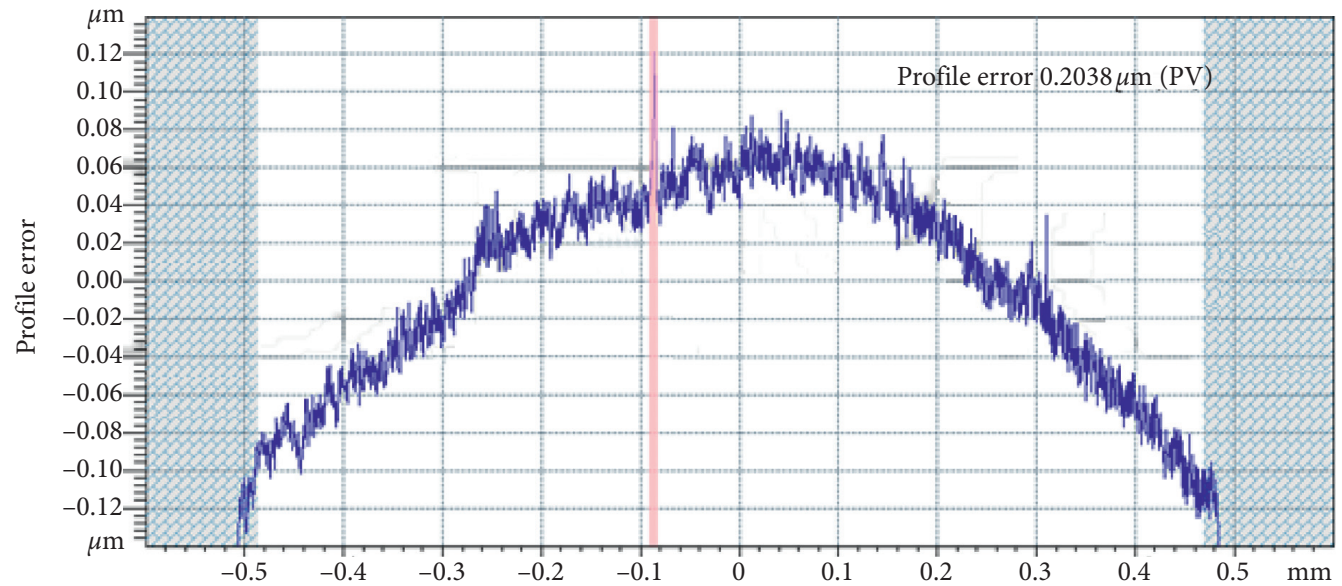

(d)

FIgURE 7: Profile error of four algorithms. (a) Profile error of RBF interpolation algorithm. (b) Profile error of four-point mean interpolation algorithm. (c) Profile error of Shepard interpolation algorithm. (d) Profile error of Hermite interpolation algorithm. 


\section{Conclusions}

This paper discusses interpolation algorithms in the tool path generation of FTS assisting diamond turning for freeform optics. Mathematical equations were built for interpolation of original data, and a positive definite RBF with compact support was employed to interpolate scattered data. Simulation and machining experiments were carried out to validate the performance of the proposed algorithm. The algorithm was found to excel in accuracy, smoothness, and error distribution in simulation experiments. Moreover, the machining results showed that the RBF interpolation algorithm can provide higher accuracy than the other three algorithms. Intervals between two cutting points should be under $0.02 \mathrm{~mm}$ in order to achieve nanolevel accuracy of interpolation. Overall, the RBF interpolation algorithm can provide higher accuracy, better smoothness, and more rational error distribution. Therefore, RBF should be employed as the interpolator for the regeneration of the surface in the tool path generation of FTS.

\section{Data Availability}

Readers can access the data underlying the findings of the study by accessing the following link: https://pan.baidu.com/ s/1SsluxhUW3_hSFTvx_23zGA, with the extracting code of Niey.

\section{Conflicts of Interest}

The authors declare that there are no conflicts of interest regarding the publication of this paper.

\section{Acknowledgments}

This study was supported by the Tianjin Application Foundation and Advanced Technology Research Program (the Youth Fund Project) under grant no. 19JCQNJC04200, the Tianjin Higher Education Science and Technology Development Fund Project under grant no. 2017KJ102, the National Natural Science Foundation of China (NSFC) under grant no. 51905378, the Tianjin Science and Technology Planning Project under grant nos. 20JCQNJC00360 and 20YDTPJC00450, the Scientific Research Foundation of Tianjin University of Technology and Education under grant no. KYQD1901, and the Open Fund of Tianjin Aerospace Intelligent Equipment Technology Key Laboratory under grant no. TJYHZN2019KT001.

\section{References}

[1] H. Ries and J. Muschaweck, "Tailored freeform optical surfaces," Journal of the Optical Society of America A, vol. 19, no. 3, pp. 590-595, 2002.

[2] J. F. Ye, L. Chen, X. H. Li, Q. Yuan, and Z. S. Gao, "Review of optical freeform surface representation technique and its application," Optical Engineering, Review, vol. 56, no. 11, p. 15, 2017.

[3] F. Duerr, P. Benitez, J. C. Minano, Y. Meuret, and H. Thienpont, "Analytic free-form lens design for imaging applications with high aspect ratio," in Current Developments in Lens Design and Optical Engineering Xiii, R. B. Johnson, V. N. Mahajan, and S. Thibault, Eds., Spie-Int Soc Optical Engineering, Bellingham, WA, USA, 2012.

[4] F. Duerr and H. Thienpont, "Optical design of static and dynamic laser beam shaping systems," in Optical Systems Design 2015: Illumination Optics Iv, T. E. Kidger and S. David, Eds., Spie-Int Soc Optical Engineering, Bellingham, USA, 2015.

[5] F. R. Fournier, "A review of beam shaping strategies for LED lighting," in Illumination Optics Ii, T. E. Kidger and S. David, Eds., Spie-Int Soc Optical Engineering, Bellingham, WA, USA, 2011.

[6] H. Li, X. Zhang, G. W. Shi, H. M. Qu, Y. X. Wu, and J. P. Zhang, "Review and analysis of avionic helmet-mounted displays," Optical Engineering, Review, vol. 52, no. 11, p. 14, 2013.

[7] F. Z. Fang, X. D. Zhang, A. Weckenmann, G. X. Zhang, and C. Evans, "Manufacturing and measurement of freeform optics," CIRP Annals, vol. 62, no. 2, pp. 823-846, 2013.

[8] H. S. Kim, K. I. Lee, K. M. Lee, and Y. B. Bang, "Fabrication of free-form surfaces using a long-stroke fast tool servo and corrective figuring with on-machine measurement," International Journal Of Machine Tools \& Manufacture, vol. 49, no. 12-13, pp. 991-997, 2009.

[9] S. Scheiding, A. Y. Yi, A. Gebhardt et al., "Freeform manufacturing of a microoptical lens array on a steep curved substrate by use of a voice coil fast tool servo," Optics Express, vol. 19, no. 24, pp. 23938-23951, 2011.

[10] F. J. Tian, Z. Q. Yin, and S. Y. Li, "Fast tool servo diamond turning of optical freeform surfaces for rear-view mirrors," International Journal of Advanced Manufacturing Technology, vol. 80, no. 9-12, pp. 1759-1765, 2015.

[11] Z. Li, F. Fang, X. Zhang, X. Liu, and H. Gao, "Highly efficient machining of non-circular freeform optics using fast tool servo assisted ultra-precision turning," Optics Express, vol. 25, no. 21, pp. 25243-25256, 2017.

[12] J. R. Kirk Rogers, "Freeform machining with precitech servo tool options," 2020.

[13] S. R. Patterson and E. B. Magrab, "Design and testing OF a fast tool servo for diamond turning," Precision Engineering, vol. 7, no. 3, pp. 123-128, 1985.

[14] S. Rakuff and J. F. Cuttino, "Design and testing of a longrange, precision fast tool servo system for diamond turning," Precision Engineering, vol. 33, no. 1, pp. 18-25, 2009.

[15] X.-D. Lu and D. L. Trumper, "Ultrafast tool servos for diamond turning," CIRP Annals, vol. 54, no. 1, pp. 383-388, 2005.

[16] D. Tong, S. C. Veldhuis, and M. A. Elbestawi, "Control of a dual stage magnetostrictive actuator and linear motor feed drive system," International Journal of Advanced Manufacturing Technology, vol. 33, no. 3-4, pp. 379-388, 2007.

[17] L. B. Kong, C. F. Cheung, and T. C. Kwok, "Theoretical and experimental analysis of the effect of error motions on surface generation in fast tool servo machining," Precision Engineering, vol. 38, no. 2, pp. 428-438, 2014.

[18] D. W. K. Neo, A. S. Kumar, and M. Rahman, "A novel surface analytical model for cutting linearization error in fast tool/ slow slide servo diamond turning," Precision Engineering, vol. 38, no. 4, pp. 849-860, 2014.

[19] D. P. Yu, G. S. Hong, and Y. S. Wong, "Profile error compensation in fast tool servo diamond turning of microstructured surfaces," International Journal of Machine Tools and Manufacture, vol. 52, no. 1, pp. 13-23, 2012. 
[20] Y. Nie, The Study of Fast Tool Servo Driven by Maxwell Normal Stress Motor and its Techniques, Tianjin University, Tianjin, China, 2014.

[21] F. Z. Fang, X. D. Zhang, and X. T. Hu, "Cylindrical coordinate machining of optical freeform surfaces," Optics Express, vol. 16, no. 10, pp. 7323-7329, 2008.

[22] Y. Hu, M. X. Xu, X. Z. Xu, Z. J. Yang, and Y. L. Zhang, "An accurate interpolator for FTS diamond turning of optical freeform surface," International Journal of Advanced Manufacturing Technology, vol. 73, no. 5-8, pp. 635-638, 2014.

[23] Q. Liu, X. Zhou, and P. Xu, "A new tool path for optical freeform surface fast tool servo diamond turning," Proceedings of the Institution of Mechanical Engineers, Part B: Journal of Engineering Manufacture, vol. 228, no. 12, pp. 1721-1726, 2014.

[24] Y. H. Nie, The Study of Fast Tool Servo Driven by Maxwell Normal Stree Motor and its Techniques, Ph.D. thesis, Tianjin University, Tianjin, China, 2014.

[25] S. Pino-Povedano, C. Bousono-Calzon, and F. J. GonzalezSerrano, "Radial basis function interpolation for signalmodel-independent localization," IEEE Sensors Journal, vol. 16, no. 7, pp. 2028-2035, 2016.

[26] R. L. Hardy, "Theory and applications of the multiquadricbiharmonic method 20 years of discovery 1968-1988," Computers \& Mathematics with Applications, vol. 19, no. 8-9, pp. 163-208, 1990.

[27] Z.-m. Wu and R. Schaback, "Local error estimates for radial basis function interpolation of scattered data," 1997.

[28] S. Rippa, "An algorithm for selecting a good parameter c in radial basis function interpolation," Advances in Computational Mathematics, vol. 11, no. 2/3, pp. 193-210, 1999.

[29] W. I. Thacker, J. Zhang, L. T. Watson, J. B. Birch, M. A. Iyer, and M. W. Berry, "Algorithm 905," Acm Transactions on Mathematical Software, vol. 37, no. 3, pp. 1-20, 2010. 